\title{
Energy Efficient Scheme for Heterogeneous Wireless Sensor Networks: Research and Challenges
}

\author{
Akshay Sharma \\ Department of Computer \\ Engineering \\ Maharishi Markandeshwar \\ University, Solan
}

\author{
Kunal Goel \\ Department of Computer \\ Engineering \\ Maharishi Markandeshwar \\ University, Solan
}

\author{
Amit Kumar Bindal \\ Department of Computer \\ Engineering \\ Maharishi Markandeshwar \\ University, Mullana
}

\begin{abstract}
Wireless Sensor Networks (WSN) are used in multiplicity arenas which embraces environmental monitoring, healthcare, battle field, biological, home, disaster management and other commercial applications. With the massive progression in the arena of embedded computer and sensor technology, Wireless Sensor Networks (WSN), that is composed of numerous thousands of sensor nodes which are proficient of sensing, actuating, and relaying the unruffled information, have made remarkable impact universally. This paper proposed the concept of energy efficient clustering for heterogeneous wireless sensor networks by the use of solar powered nodes. Furthermore the configurability of sensor nodes in instance of any disaster occur, as well as propositioning a new sensor node, which will make the network reliable and increase the lifespan of the network.
\end{abstract}

Keywords- Cast-off, Vitality, Spawn, Li-ion, Energy Harvesting Tails, Instance, Perishes.

\section{INTRODUCTION}

Wireless sensor networks entails the number of sensor nodes that are position in environs which are cast-off to sense the numerous influences like sound, temperature, pressure, vibration, etc. The sensor nodes can be positioned in numerous certain areas where they are not effortlessly accessible so, to bar the vitality of the sensor node is foremost challenge of the wireless sensor networks [1]. As we know that the life span of communication network in WSN is limited, so to increase life span of the network, energyefficient protocols should be the prime aim [2]. Hierarchicalbased routing technique is based on clustering in which the sensor nodes with high energy level are casually selected for processing and sending information to the Base Station (BS), while the sensor nodes with low energy level are only used for sensing the data from the sensing filed. Clustering makes the performance of sensor network more efficient and which increases the lifetime of the network [3]. As we requisite to depend upon the hardware, so we will use Li-ion [14] battery powered sensor nodes as their self-discharge rate is much slower as compared to other batteries. As the table1 shows, the Li-ion battery is the utmost reliable technology for the wireless communication generally due to their greater energy and power density as paralleled to Nickel Cadmium and Nickel Metal Hydride [4]. Another approach to resolution of problem is the energy harvesting method, which spawn the vitality by itself. Energy harvesting means to harness the energy from ambient energies like heat energy, wind energy, tidal energy, steam energy and convert into the electrical energy for sensor batteries. Broadly, Energy Harvesting can be divided into two architectures-(i) Harvest-Use; Energy is harvested just in time for use (ii) Harvest-Store-Use. Energy is harvested when conceivable and stored for future use [5]. The table given below is showing the comparison of different batteries.

Table 1. Comparisons of various batteries used in Sensor Nodes

\begin{tabular}{|l|l|l|l|}
\hline & Li-ion & NiCad & NiMH \\
\hline $\begin{array}{c}\text { Energy Density } \\
(\text { Wh/Kg) }\end{array}$ & $100+$ & 50 & 70 \\
\hline $\begin{array}{l}\text { Leakage } \\
(\% / M o n t h)\end{array}$ & 8 & 15 & 30 \\
\hline $\begin{array}{l}\text { Operating } \\
\text { voltage }\end{array}$ & 3.6 & 1.2 & 1.2 \\
\hline
\end{tabular}

\section{LITERATURE REVIEW}

Clustering in wireless sensor networks is classified into two types of schemes, homogeneous and heterogeneous schemes. In homogeneous the initial energy is same for all the nodes, while in case of heterogeneous networks there is a change in initial energy level of sensor nodes. LEACH, HEED, PEAGSIS are some examples of the homogeneous networks but they do not behave well in case of heterogeneous networks. In instance of homogeneous systems the hierarchical clustering algorithm is proposed which is named as LEACH (Low Energy Adaptive Clustering Hierarchy) which allocates the local cluster as practice of base stations for stable dispersal of energy between sensors [6]. In HEED protocol the assortment of cluster head is ended upon periodic based on hybrid of their unconsumed vitality and sensor node propinquity to its fellow sensor node [7]. The PEAGSIS protocol is the chain based protocol which pacts with communiqué with adjacent sensor nodes and ensues turns communicating to the sink [8]. For heterogeneous wireless sensor networks there are different types of models of energy levels: two level, three and multi-level energy model [13].

\subsection{Two Energy Level Heterogeneous Model of WSN}

In two level heterogeneous model of Wireless Sensor Network, it comprehends of two types of sensor nodes with respect to their energy level, normal nodes and advanced nodes [15]. The energy for the normal node is represented by 
Eo. Although the energy for the advanced sensor node is denoted by $E o(1+a)$, where $a$ signifies that the advanced contains a times supplementary energy as related to normal nodes. $\mathrm{N}$ refers to the number of total nodes where $\mathrm{m}$ refers to the fraction of the advanced nodes. The overall energy of the network is the sum of the energies of the normal and advanced nodes.

Etotal $=N(1-m) E o+N m(1+a) E o$

$=N E o(1-m+m+a m)$

$=N E o(1+a m)$

\subsection{Three Energy Level Heterogeneous Model of WSN}

In three level heterogeneous model of Wireless Sensor Network, it comprehends of three types of sensor nodes with respect to their energy level, normal advanced and super nodes. Eo represents the energy of the normal node where $E o(1+a)$ signifies the energy level of advanced nodes. Whereas advanced nodes having a times more energy than normal nodes. And $E o(l+b)$ signifies the energy level of Super nodes. Whereas super nodes having $b$ times more energy than normal nodes. The total energy of the three level heterogeneous WSN network is given by:

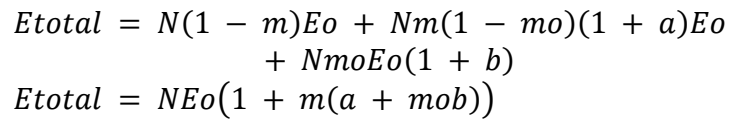

\subsection{Multi-level Heterogeneous Model of WSN}

In multilevel heterogeneous model of Wireless Sensor Network, it comprehends of multi-types of sensor nodes with respect to their energy level. The preliminary energy of nodes is circulated above the close set that is $[E o, E o(1+\operatorname{amax})]$, where $E o$ is the value of the lower energy level and amax is the value of maximum energy. The total energy of the multilevel heterogeneous WSN network is given by:

Etotal $=\sum_{i=1}^{N} E o(1+a i)=E o\left(N+\sum_{i=1}^{N} a i\right)$

In SEP, [9] protocol for heterogeneous networks to lengthen the time interim afore the passing of the leading sensor node. SEP is grounded on based of selection prospects of each sensor node to seizure into cluster head bestowing to the lasting vitality in respectively sensor node. But the foremost downside of SEP is that it can merely act well for just two glassy of heterogeneity but in instance of multi-level heterogeneity implementation of SEP is contemptible. The [2] DEEC (Distributed energy efficient Clustering) algorithm proposed the scheme in which cluster heads are designated on the prospect grounded on fraction of the residual energy of respectively sensor node and the unexceptional energy of the network. The cluster head establishment is grounded on the high preliminary energy of the sensor nodes. The sensor nodes with squat level of vitality have no probabilities to become the cluster head. In DEEC there are two natures of sensor nodes whose energy level is dissimilar, normal and advance. To protect energy DEEC practices the despicable energy as allusion energy. The prospects of the assortment the cluster head by spending two echelons of nodes is defined by this equation: $p i=\left\{\begin{array}{l}\frac{\text { poptEi(r) }}{(1+a m) \bar{E}_{(r)}}, \text { if si is the normal node } \\ \frac{\text { popt(1+a)Ei(r) }}{(1+a m) \bar{E}_{(r)}}, \text { if si is the advanced node }\end{array}\right.$

Here are the various considerations that the researcher practices in DEEC protocol:

\section{Table 2: Parameters Used in DEEC Protocol}

\begin{tabular}{|l|l|}
\hline \multicolumn{1}{|c|}{ Parameter } & \multicolumn{1}{c|}{ Value } \\
\hline Eelec & $5 \mathrm{~nJ} / \mathrm{Bit}$ \\
\hline Efx & $10 \mathrm{pJ} / \mathrm{Bit} / \mathrm{m}^{2}$ \\
\hline Emp & $0.0013 \mathrm{pJ} / \mathrm{Bit} / \mathrm{m}^{4}$ \\
\hline Eo & $0.5 \mathrm{~J}$ \\
\hline Eda & $5 \mathrm{~nJ} / \mathrm{Bit} / \mathrm{Message}$ \\
\hline Do & $70 \mathrm{~m}$ \\
\hline Message size & $4000 \mathrm{bits}$ \\
\hline Popt & 0.1 \\
\hline
\end{tabular}

In [10] distributed energy-efficient clustering for heterogeneous wireless sensor networks the cluster head selection prospect is grounded on the dynamic alteration and with additional effectiveness. E-DEEC, [3] a protocol in which there are three categories of energy level sensor nodes: normal, advance and super nodes. By captivating super node it will protract the lifecycle of the system and accomplish thriving for multi-level heterogeneous Communication. The likelihoods of the assortment of the cluster head by expending three altitudes of nodes are defined by the below equations:

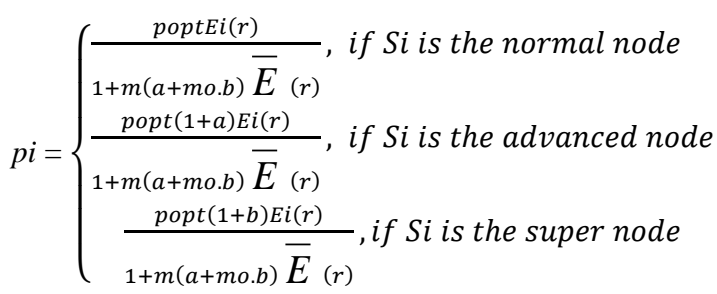

EDDEEC, [11] a protocol that custom a pattern in which the prospect of mutable the Cluster Head is dynamic and it will clearance the equivalent volume of energy amongst the sensor nodes. BEENISH, [12] (Balanced Energy Efficient Network Integrated Super Heterogeneous) protocol proposed the four unalike levels of sensor nodes: Normal, advance, Super, Ultra super. In BEENISH first sensor node perishes after 1103 round and last sensor node perishes after 6903 round. In BEENISH there are four diverse varieties of energy level nodes are there and the prospect of medley of the cluster head is demarcated by this equation:

$p i=$

$$
\left\{\begin{array}{l}
\frac{p o p t E i(r)}{1+m(a+m o(-a+b+m 1(-b+u))) \bar{E}(r)}, \text { si is the normal node } \\
\frac{p o p t(1+a) E i(r)}{1+m(a+m o(-a+b+m 1(-b+u))) \bar{E}(r)}, \text { si is the advanced node } \\
\frac{p o p t(1+b) E i(r)}{1+m(a+m o(-a+b+m 1(-b+u)))} \bar{E}_{(r)} \\
\frac{p o p t(1+u) E i(r)}{1+m(a+m o(-a+b+m 1(-b+u)))} \text {, si is the super node }
\end{array}\right.
$$




\section{CHALLENGES}

There are some issues that occur in aforementioned version of heterogeneous protocols. In DEEC, EDEEC, BEENISH they all are haphazardly selected the cluster head based on its residual energy level and after some time the energy level of super or ultra-super nodes is equal to the energy level of the normal nodes. Due to this the energy level of the network goes down.Secondly, BEENISH runs up to 6903 rounds after that all nodes dead so lifespan of the network is short.

\section{PROPOSED MODEL}

\subsection{Sensor Node Used in WSN}

The design of Prometheus entails of Lithium rechargeable batteries. The amount of leakage of lithium batteries is lowest with high densities and comprises high power with a solo cell. Lithium batteries are far superior then NiMH batteries to operate with narrow recharge cycles. In case of lithium batteries, they need to be charged from primary buffer where energy is constant. The solar panel that is using for Prometheus yields $4.5 \mathrm{~V}$ and contests the $5 \mathrm{~V}$ extreme voltage of primary buffer. The two super capacitors that are using are Aerogel because of their small leakage value. They are using a voltage of $2.5 \mathrm{~V}$ each. The power of the sensor node is dependent upon three factors: duty cycle, active mode and sleep mode. The average power consumption is:

$$
P c A V G=\text { Vsupply } *(D * \text { Iactive }+(1-D) * \text { Isleep })
$$

Prometheus consists of lithium batteries, solar panel $(37 \times 82 \mathrm{~mm})$, two super capacitors, and telos sensor node as shown in figure1:

If the telos sensor node operates on $1 \%$ duty cycle, then the average power consumption is:

$$
\frac{20 m A+99 * 5 u A}{100}=205 u A
$$

Beneath no light occurrence the output of the system (200mAh battery) is mentioned below:

$$
\frac{0.2 A h}{\left(205 * 10^{-6 A}\right) * 24 \text { hours }}=40.65 \text { days }
$$

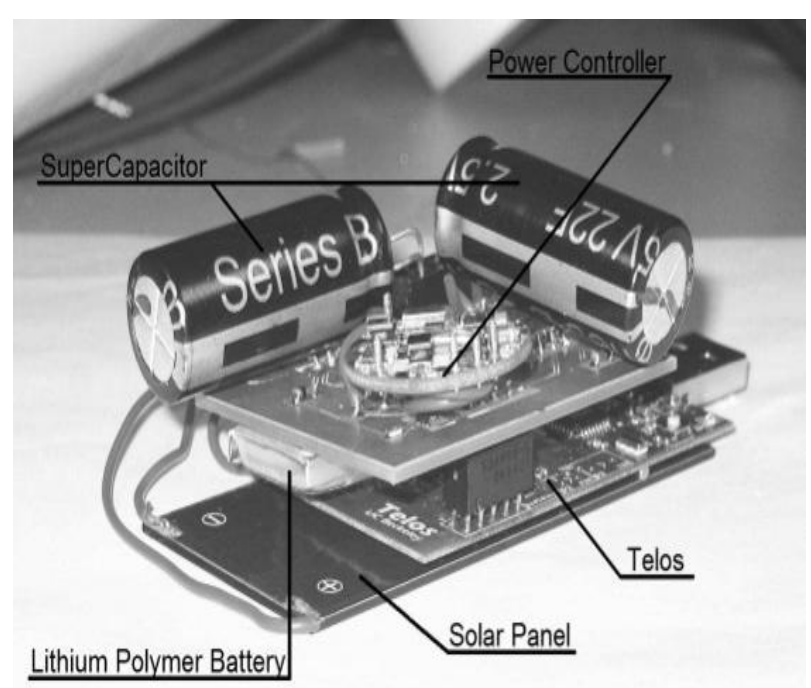

Figure1: Prometheus, Solar harvesting sensor node for wireless sensor network
A superior battery results in unvaryingly elongated time. This is the chief intention that we are using Prometheus for our network just because of the fact if there is no sunlight for continuous 40 days it can be operate and this can be longer depends upon the duty cycle. In case of the wireless sensor networks the duty cycle is $1 \%$ or less than one percent. The foreseen era of Lithium battery and Super capacitors according to node duty cycle is shown in table3.

TABLE3. PREDICTED LIFESPAN AND EFFECT OF NODE DUTY CYCLE

\begin{tabular}{|l|l|l|}
\hline Duty Cycle & Required Light & Lifetime \\
\hline $1 \%$ & $5 \mathrm{hr} / \mathrm{month}$ & 43 years \\
\hline $10 \%$ & $5 \mathrm{hr} / 4$ days & 4 years \\
\hline $100 \%$ & $10 \mathrm{hr} / 1$ day & 1 year \\
\hline
\end{tabular}

\subsection{System Model}

In our proposed model as shown in figure 2 the network consists of the random sensor nodes deployed in the field. The sensor nodes start sending their energy level, location and their respective id to the base station. The sensor nodes with rechargeable batteries are considered to be selected as cluster heads. In this network model we had taken the five different types of nodes. Normal, Advanced, Super, Ultra Super, and Ultra plus nodes with various energy levels. In some nodes we are deploying the technique of energy harvesting that harvest the energy from solar and convert it into the electrical energy for the Lithium battery used in sensor node. A random deployment of the sensor nodes in the network is shown in figure2.

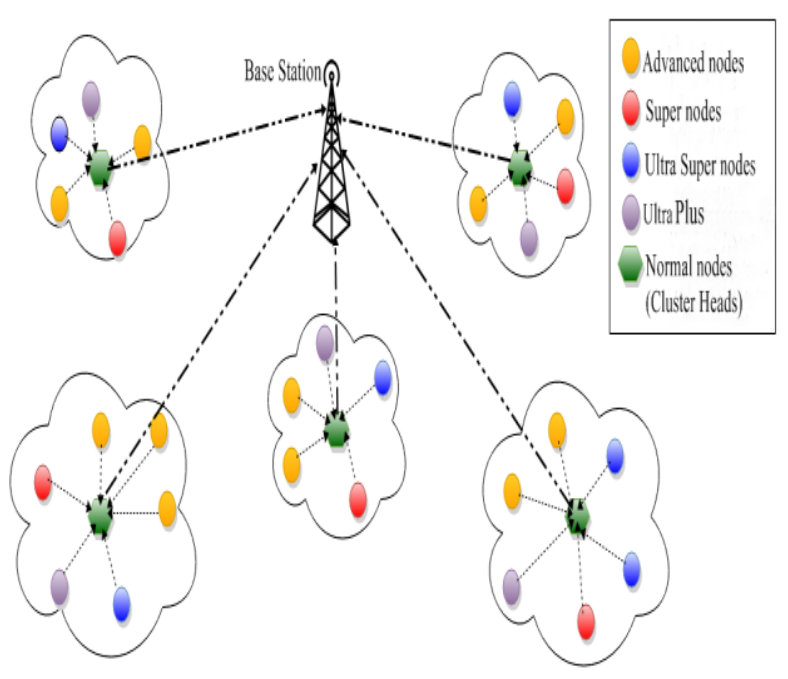

Figure 2: Model for Energy Efficient Wireless Sensor Networks

In our proposed system model if there is any case any sensor failure occur then sensor network call the recursive algorithm to rearrange it. If there is no fiasco of sensor node befalls the sensors starts sensing the data and transmits the sensed data to their respective cluster heads. The cluster heads sends the totaled data to the base station. Below is the flow diagram of the network model as shown in figure 3 . 


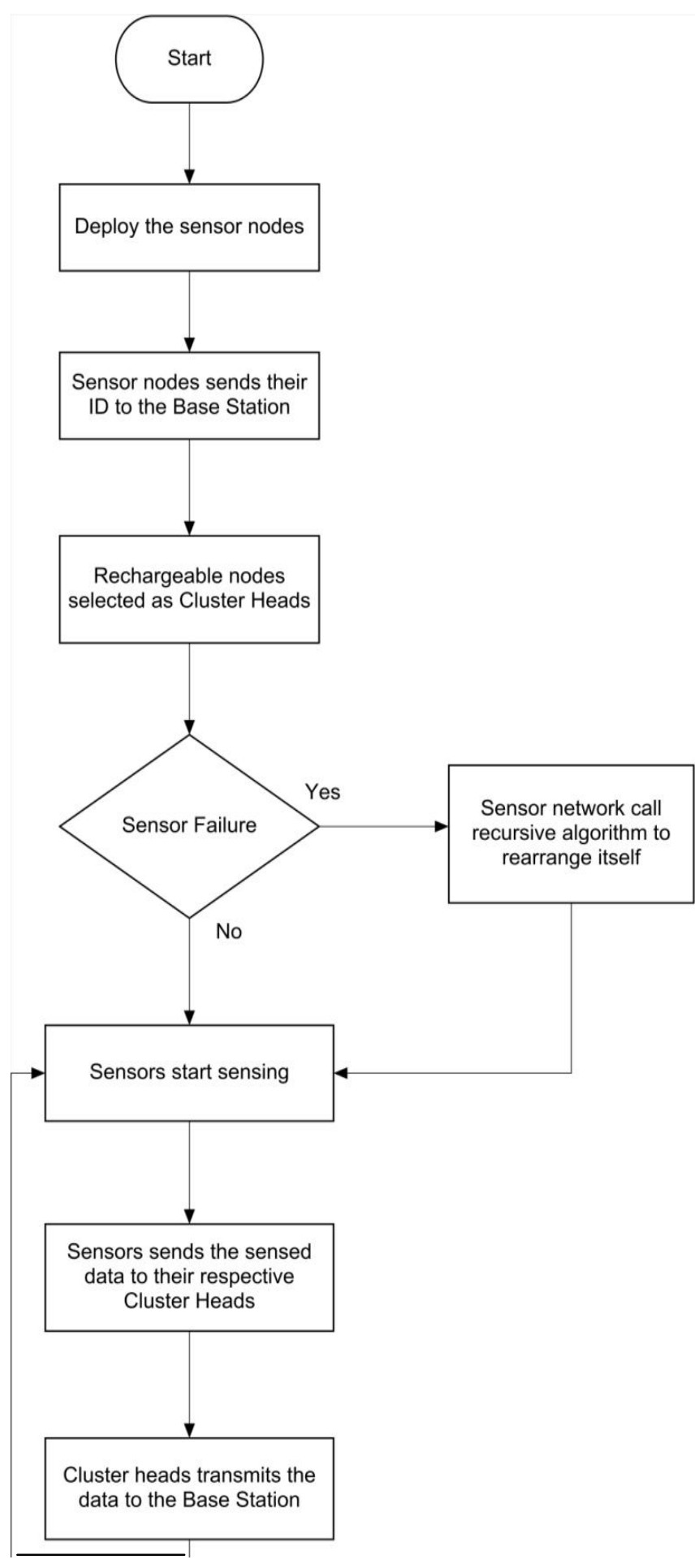

Figure 3: Heterogeneous node management \& Communication System.

\subsection{Energy model}

In our proposed scheme we are using the concept of rechargeable batteries via solar energy on some nodes. As we are using the concept of solar energy on some nodes, every time the battery level of the those nodes (selected as cluster heads) reaches to the critical point it will send signal to base station for low energy level. Base station transmits that signal to open the charging circuit. $\mathrm{CH}$ will open the charging circuit and charge the battery via solar energy. Here is the flow diagram of the energy model:

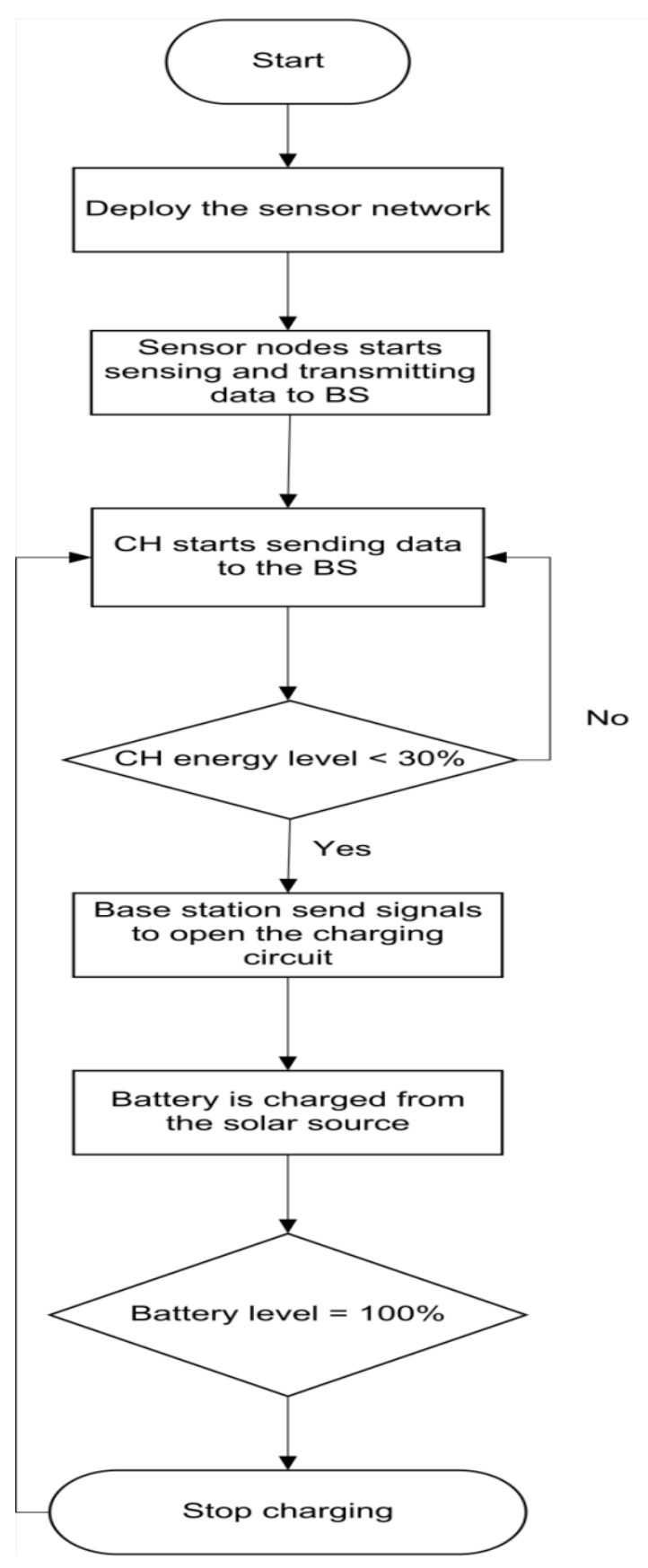

Figure4: Reliable and Efficient Energy Model

\section{CONCLUSION}

The author in this paper concludes about the hierarchy of sensor nodes and solar powered sensor nodes (Prometheus) used in wireless sensor network. In this the author had taken the five different types of energy level sensor nodes in a form of clustering. The sensor nodes transmitting the data to their respective cluster heads and further they are transmitting the data to base station. Second we analysis the various energy cells for sensor nodes and the author finds the Lithium is trustworthy. Third thing is to afford the elongated existence of network. The author uses the concept of energy harvesting in the network model. 


\section{FUTURE WORK}

In Future the author have to increase the number of sensor nodes and the deployment area and to analyze the performance to reduce the delay in the network. Analysis can be done on how much solar energy is required for Li-Ion cell so it can communicate the data packet to base station without any delay and there should be no communication failure occur in respect of energy. We will have to analyze the parameters that are required for the transformation of solar energy into electrical energy.

\section{REFERENCES}

[1] Tauseef Shah, Nadeem Javaid, Talha Naeem Qureshi, "Energy Efficient Sleep Awake Aware (EESAA) Intelligent Sensor Network Routing Protocol", $15^{\text {th }}$ IEEE International Multi Topic Conference (INMIC'12) 2012, Pakistan.

[2] L. Qing, Q. Zhu, M. Wang, "Design of a distributed energy-efficient clustering algorithm for heterogeneous wireless sensor networks", ELSEVIER Computer Communication, PP: 2230-2237, 2006.

[3] Parul Saini, Ajay K Sharma, "E-DEEC - Enhanced Distributed Energy Efficient Clustering Scheme for Heterogeneous WSN", IEEE $1^{\text {st }}$ International Conference On Parallel, Distributed and Grid Computing, (PDGC2010).

[4] Waleed Hadi Madhloom Kurdi and Amit Bindal, "Effective Implementation of Power Optimization in Wireless Sensor Networks", IJECBS, Systems, Vol. 4 Issue 2, July 2014.

[5] Sujesha Sudevalayam and Purushottam Kulkarni, "Energy Harvesting Sensor Nodes: Survey and Implications", IEEE Communications Surveys \& Tutorials, Vol 13, No 3, Third Quarter 2011.

[6] W.Heinzelman, A. Chandrakasan, and H. Balakrishnan, "Energy-efficient communication protocol for wireless sensor networks," in the Proceeding of the Hawaii International Conference System Sciences, Hawaii, January 2000.

[7] O. Younis, S. Fahmy, "HEED: A hybrid, energy efficient, distributed clustering approach for adhoc sensor networks", IEEE Transactions on Mobile Computing vol 3, no 4, pp 660-669, 2004.
[8] S. Lindsey and C. S. Raghavendra, "PEGASIS: Power Efficient Gathering in Sensor Information Systems," in the Proceedings of the IEEE Aerospace Conference, Big Sky, Montana, March 2002

[9] G. Smaragdakis, I. Matta, A. Bestavros, "SEP: A Stable Election Protocol for clustered heterogeneous wireless sensor networks", in: Second International Workshop on Sensor and Actor Network Protocols and Applications (SANPA 2004), 2004.

[10] Elbhiri B., Saadane, R., El Fkihi, S., Aboutajdine, D., "Developed Distributed Energy-Efficient Clustering (DDEEC) for heterogeneous wireless sensor networks", in: 5th International Symposium on I/V Communications and Mobile Network (ISVC), 2010.

[11] N. Javaid, T. N. Qureshi, A. H. Khan, A. Iqbal, E. Akhtar, M. Ishfaq, "Enhanced Developed Distributed Energy-Efficient Clustering for Heterogeneous Wireless Sensor Networks", Procedia Computer Science ELSEVIER, International Workshop on Body Area Sensor Networks (BASNet-2013).

[12] T. N. Qureshi, N. Javaid, A. H. Khan, A. Iqbal, E Akhtar, M. Ishfaq , "BEENISH: Balanced Energy Efficient Network Integrated Super Heterogenous Protocol for Wireless Sensor Networks", in: Procedia Computer Science ELSEVIER, International Workshop on Body Area Sensor Networks (BASNet-2013).

[13] Qureshi, T.N., Javaid, N., Malik, M., Qasim, U., Khan, Z.A., "On Performance Evaluation of Variants of DEEC in WSNs", Seventh International Conference on Broadband, Wireless Computing, Communication and Applications (BWCCA), 2012, pp.162,169, 12-14 Nov. 2012.

[14] Xiaofan Jiang, Joseph Polastre, David Culler, "Perpetual Environmentally Powered Sensor Networks", in Fourth International Symposium on Information Processing in Sensor Networks., April 2005, pp. 463-468.

[15] Amit Kumar Bindal, Ankur Mangla, Devendra Prasad, R. B. Patel, "P LDP: Possible Location Deployment Protocol for Energy Harvesting in WSNs", 2nd IEEE sponsored International conference on Parallel, Distributed and Grid Computing (PDGC 2012) paper ID 1569664227,December 6-8,2012 Jaypee University of Information Technology, Waknaghat, Solan-173234, Himachal Pradesh, INDIA. 